\title{
Level of Block
}

\author{
Charlene Pius ${ }^{1}$, Wern Yew Ding ${ }^{1}$, Saagar Mahida ${ }^{1}$, and Simon Modi ${ }^{2}$ \\ ${ }^{1}$ Liverpool Heart and Chest Hospital NHS Trust \\ ${ }^{2}$ Liverpool Heart and Chest Hospital
}

June 17, 2020

\section{TITLE PAGE}

TITLE : LEVEL OF BLOCK

DESCRIPTION :

Electrograms recorded from an electrophysiological study and ablation of manifest left lateral accessory pathway with demonstration of site of block during the case.

\section{AUTHORS}

Dr. Charlene Pius (corresponding author)

MBChB, MRCP (UK) Liverpool Heart \& Chest Hospital, Thomas Drive, Liverpool, L14 3 PE UK

cpius217@gmail.com

Dr. Ding Yew Wern

MBChB, MRCP (UK)

Liverpool Heart \& Chest Hospital, Thomas Drive, Liverpool, L14 3 PE UK

Dr. Saagar Mahida

MD, PhD Liverpool Heart \& Chest Hospital, Thomas Drive, Liverpool, L14 3 PE UK

Dr. Simon Modi

MBBS, MRCP Liverpool Heart \& Chest Hospital, Thomas Drive, Liverpool, L14 3 PE UK

No conflicts of interests to declare.

No funding

A 27 year old patient underwent electrophysiology testing and catheter ablation for a manifest left lateral accessory pathway (AP). The following electrograms were recorded during electrophysiology testing of a manifest left lateral accessory pathway (AP). Bidirectional pathway conduction was seen and orthodromic atrioventricular re-entrant tachycardia was induced with premature atrial stimuli and anterograde pathway block. Through a transseptal approach, mapping of the mitral annulus (3 o'clock position) produced Figure 1. Stabilization of the catheter, ready for radiofrequency ablation, produced figure 2 . Where is the level of block?

\section{DISCUSSION}

Figure 1 demonstrates mapping of the pathway ventricular insertion during atrial pacing $(600 \mathrm{~ms})$ from distal coronary sinus (dCS). Two beats conduct with pre-excitation and 2 without. HV intervals during pre-excited 
beats are negative as expected for a left lateral accessory pathway differentially paced from dCS. A balanced atrial and ventricular signal is seen on ablation distal (ABLd). A separate, high frequency electrogram, dividing the atrial and ventricular electrograms and distinct from His timing is seen, likely representing a pathway potential. Catheter pressure at this site caused intermittent loss of pre-excitation. The non-preexcited beats continue to demonstrate the pathway potential confirming that the level of block falls at its ventricular insertion point.

Figure 2 similarly demonstrates intermittent pre-excitation. With increased catheter pressure during stabilization immediately prior to ablation, one not only appreciates block at the ventricular insertion but also now variable conduction at the atrial insertion point.

Radiofrequency ablation at this site abolished the pathway potential and pre-excitation. Tachycardia thereafter was non-inducible.

Calkins et al have previously demonstrated that the site of block during an accessory pathway (AP) ablation, irrespective of mapping techniques, was more commonly located between the atrial electrogram and the pathway potential $(78 \%)$ with only $9 \%$ of patients demonstrating block between the pathway potential and the ventricular insertion point ${ }^{1}$. However, Ueng et al demonstrated a paradox in the site of block during AP ablation. Despite commonly mapping the earliest atrial activation in concealed APs, they curiously demonstrated a higher incidence of block ultimately between the pathway potential and the ventricular insertion point (70.9\%). They found the converse true for manifest AP ablation ${ }^{2}$. We believe our case is the first published case clearly demonstrating simultaneous block at both the ventricular and atrial insertion sites during accessory pathway ablation.

\section{REFERENCES}

1. Calkins H, Mann C, Kalbfleisch S, Langberg JJ, Morady F. Site of accessory pathway block after radiofrequency catheter ablation in patients with the Wolff-Parkinson-White syndrome. J Cardiovasc Electrophysiol . 1994;5(1):20-27. doi:10.1111/j.1540-8167.1994.tb01111.

2. Ueng, K.-C., Chen, S.-A., Chiang, C.-E., Cheng, C.-C., Wu, T.-J., Tai, C.T Chang, M.-S. (1996). Paradox of Accessory Pathway Block After Radiofrequency Ablation in Patients with the Wolff-ParkinsonWhite Syndrome. Angiology , 47 (11), 1061-1071.

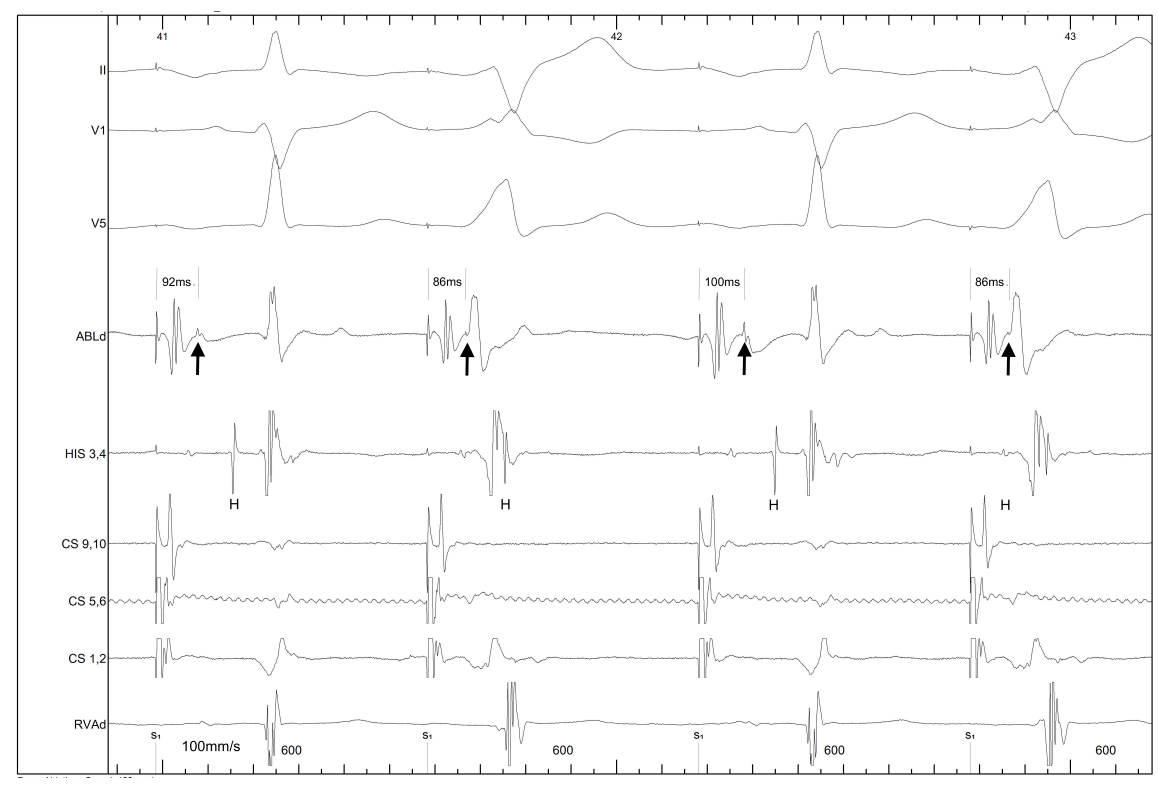




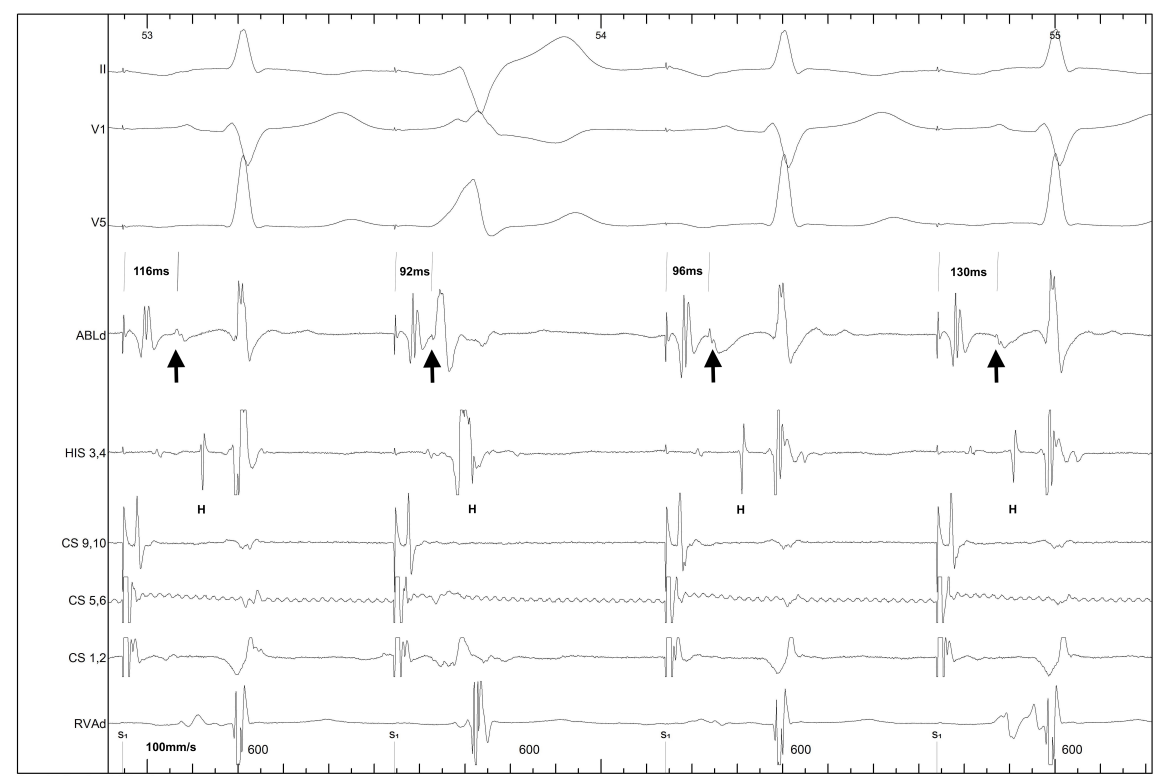

